Bangladesh J. Plant Taxon. 14(1): 25-35, 2007 (June)

\title{
A TAXONOMIC REVISION OF THE GENUS CLEOME L. (CAPPARACEAE) IN BANGLADESH
}

\author{
Hosne Ara ${ }^{1}$, Bushra Khan and Md. Manzur-Ul-Kadir Mia \\ Bangladesh National Herbarium, Chiriakhana Road, Mirpur 1, \\ Dhaka 1216, Bangladesh
}

Key words: Cleome, Taxonomic revision, Capparaceae, Bangladesh

\begin{abstract}
The available record of the genus Cleome L. for Bangladesh has been updated in this paper and includes C. diffusa, C. gynandra, C. hassleriana, C. rutidosperma and C. viscosa. The updating has been done in case of nomenclature with important synonyms, local names, description of the taxa along with illustrations, flowering and fruiting times, ecological notes, specimens examined and their geographical distribution. For identification of the species, a dichotomous bracketed key has been added in this paper. Information on chromosome number and economic importance have also been provided where available.
\end{abstract}

\section{Introduction}

The genus Cleome was first described by Linnaeus in Species Plantarum, Vol. 2 in 1753. The genus is represented by more than 150 species in pantropical and subtropical regions of the world (Jacobs 1960). Many of them in America, in the Old world (c. 65 spp.), mostly in Africa and the Middle East. There are eight species in Malaysia, of which two are cultivated and the others are native or introduced. Many species have been introduced into other continents as aliens and are widely spread as weeds (Jacobs 1960). There are about 15 species in India (Raghavan 1993).

Taxonomic revision of the genus Cleome L. in Bangladesh has not been done. Hooker and Thomson (1872) mentioned 12 species of Cleome L. and one species of Gynandropsis, G. pentaplylla which is now treated as Cleome gynandra, from British India. Prain (1903) recorded only five species including the species of Gynandropsis for the greater Bengal of which only two fall in the territory of Bangladesh. Khan et al. (1978) recorded two species of Cleome, viz. C. rutidosperma and C. hassleriana for Bangladesh. Yusuf (1989) also recorded one species of Cleome, C. diffusa, for Bangladesh. A literature survey including Heinig (1925), Kanjilal et al. (1934), Datta and Mitra (1953), Sinclair (1955), Khan and Banu (1972), Khan et al. (1994), Mia and Khan (1995), Rahman and Hassan (1995), Rahman and Uddin (1997), Uddin et al. (1998), Rashid et al. (2000), Rahman (2004a, 2004b) and the study of herbarium materials of Bangladesh National Herbarium (DACB) and Dhaka University Herbarium (DUH) reveals that only five species have so far been reported from Bangladesh, viz. Cleome

${ }^{1}$ Corresponding author. E-mail: bnh_mirpur@yahoo.com 
diffusa, C. gynandra, C. hassleriana, C. rutidosperma and C. viscosa. The present paper updates the available record of the genus Cleome for Bangladesh and includes important synonyms, descriptions of the taxa with illustrations, flowering and fruiting times, ecological notes and geographical distribution.

Cleome L., Sp. Pl. 2: 671 (1753).

Polanisia DC., Prod. 1: 242 (1824).

Gynandropsis DC., Prod. 1: 237 (1824). Lectotype species: C. ornithopodioides L.

Annual or perennial herbs, sometimes woody at base, often hairy, sometimes glandular-hairy, some thorny. Stipules absent or sometimes with short spine-like (thorn). Leaves alternate, long-petiolate, palmately compound; leaflets 3-9, sessile. Flowers bisexual; white, yellow, pink or purple in terminal, bracteate racemes, actinomorphic or zygomorphic by displacement of petals. Sepals generally 4, free or connate at base, equal, valvate, often glandular pubescent. Petals usually 4, mostly clawed at base, hypogynous. Androgynophore present or not. Stamens 4 to numerous, usually all fertile, borne on a short or long androgynophore; filaments equal or subequal, declinate. Ovary 1-celled, sessile or mostly on a short gynophore which elongates in fruit; ovules many, borne on 2 parietal placentas; style short or 0, stigma knob-shaped or flattish or discoid or capitate, subsessile. Fruit a capsule, capsule siliquliform, linear, terete, striate, 2-valved, beaked, dehiscing from the base with a persistent replum. Seeds numerous, glabrous or pubescent, orbicular to reniform, scalariform with conspicuous, \pm sharp cross-ribs connected by numerous, much lower longitudinal ribs or verrucose.

\section{Key to the species:}

1 Androgynophore present $\quad 2$

- $\quad$ Androgynophore absent $\quad 3$

2 Gynophore 1-2 cm long C. gynandra

- $\quad$ Gynophore 3-7 cm long $\quad$ C. hassleriana

3 Flowers white; gynophore c. $2 \mathrm{~mm}$ long $\quad$ C. diffusa

- $\quad$ Flowers yellow or pink or purplish; gynophore present or absent 4

$4 \quad$ Flowers yellow; stamens 10-24; gynophore absent

C. viscosa

- $\quad$ Flowers pink or purplish; stamens 6; gynophore 1.5-8 mm long

C. rutidosperma

1. Cleome diffusa Banks ex DC., Prodr. 1: 241 (1824). Eichl. in Mart., Fl. Bras. 13: 258 (1865); Yusuf, J. Asiatic Soc. Bangladesh (Sc.) 15 (2): 149-151 (1989).

(Plate 1)

English name: Spreading spider flower.

Erect, annual bushy herb, up to $70 \mathrm{~cm}$ high. Stem cylindric. Leaves palmately compound; leaflets 3-5, subsessile, lanceolate to elliptic-lanceolate, acute, entire, the middle one largest up to $5.7 \times 2.2 \mathrm{~cm}$, the lateral two medium up to $4.5 \times 1.9 \mathrm{~cm}$ and the 
outermost two shortest up to $3 \times 1.8 \mathrm{~cm}$. Petiole $6 \mathrm{~cm}$ long, subtended by two stipular spines. Racemes terminal, $30 \mathrm{~cm}$ long. Bracts ovate, acute to acuminate, base rounded. Pedicel 1-1.2 cm long, pubescent. Sepals 4, 3-4.5 mm long, linear-lanceolate, valvate. Petals 4, $6.5 \times 2.5 \mathrm{~mm}$, free, white. Stamens 6, the 4 anterior c. $8.5 \mathrm{~mm}$ long while the 2 posterior ones c. $7.5 \mathrm{~mm}$ long, free; anthers $1.5 \mathrm{~mm}$ long, linear, oblong, basifixed, dehiscence longitudinal. Ovary 4.5-6.5 mm long, oblong cylindric on a gynophore c. 2 mm long; ovules many, on parietal placentae; style 0; stigma capitate. Capsule up to $2.5 \times$ $0.4 \mathrm{~cm}$, elongated cylindric, apex notched, pedicel c. $1.6 \mathrm{~cm}$ long. Seeds many, 18-24, c. $2 \mathrm{~mm}$ across, rugose, strophiole white, brown, prominent. Flowering and fruiting: AprilAugust.

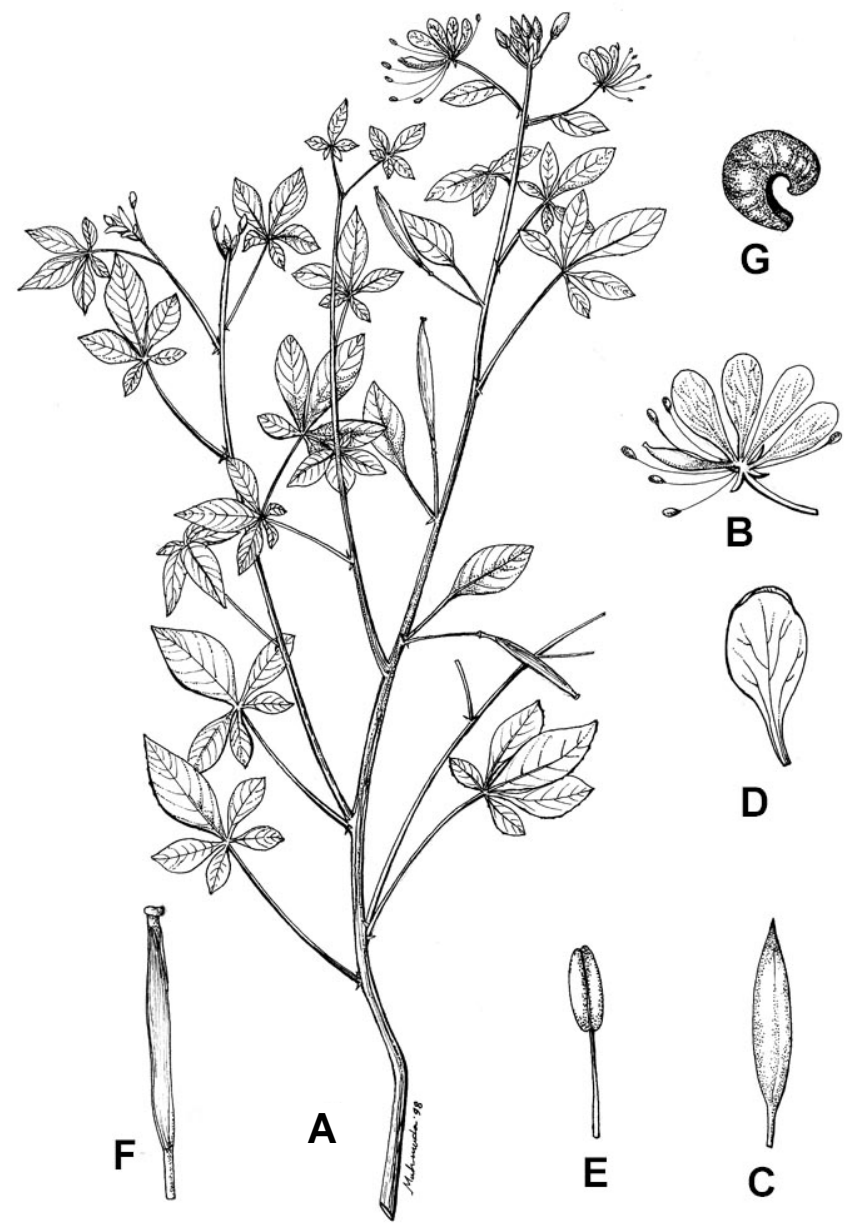

Plate 1. Cleome diffusa. A. habit (× 0.33); B. flower (× 1.67); C. sepal (× 5); D. petal $(\times 3)$; E. stamen $(\times 2.5)$; F. fruit $(\times 1)$; G. seed $(\times 5)$.

Ecology: Grows in shady damp waste places. 
Specimens examined: Chittagong: Chandanpura, 30 v 1978, Yusuf 165 (DACB); Chittagong Govt. College, 18 vii 1978, s.n. (DACB).

Geographical distribution: Brazil (type).

2. Cleome gynandra L., Sp. Pl. 2: 671 (1753). Jacobs, Fl. Males. 1 (6): 101 (1960); Grierson \& Long, Fl. Bhut. 1 (2): 416 (1984); Raghavan in Balakrishnan (ed.), Fl. Ind. 2: 309 (1993); Gynandropsis pentaphylla DC., Prod. 1: 238 (1824); Hook. f. \& Thoms., Fl. Brit. Ind. 1: 171 (1872); Prain, Beng. Pl. 1: 225 (1903), Rep. ed. 1: 150 (1963); Kanjilal et al., Fl. Assam 1: 73 (1934).

(Plate 2)

Bangla names: Sada hurhuria, Ansarisha, Arkahuli

English names: Spider wisp, Wild spider flower.

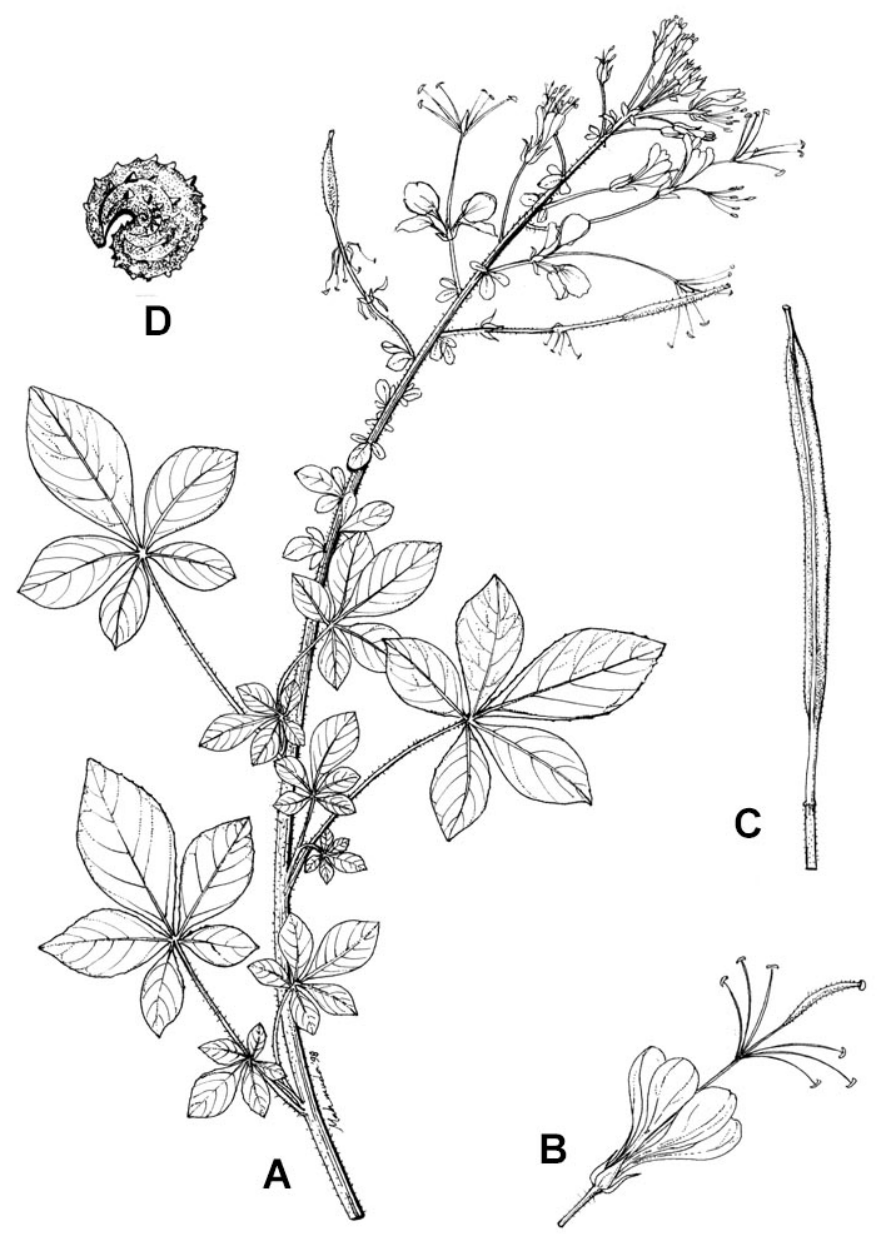

Plate 2. Cleome gynandra. A. habit (× 0.4); B. flower $(\times 1)$; C. fruit $(\times 1)$; D. seed $(\times 8)$.

Annual herb, erect, branched, 0.6-1.0 m high; all parts glandular pubescent. Leaflets 5, 0.7-5 $\times$ 0.3-3 cm, obovate-oblanceolate, lowest pair smallest, middle largest, apex 
acute or obtuse, base cuneate or attenuate, margins entire or serrulate; nerves 5-9 pairs. Petiole 3-8 cm long. Bracts small, leaf-like, trifoliate. Racemes terminal, elongated, glandular-pubescent, flowering at the tip. Flowers white or tinged with purple. Pedicels 1-2 cm long. Sepals 4, 3-6 × 1-2 mm, free, lanceolate, glandular-pubescent, green with white veins. Petals 4, 7-15 mm long in all, 1.5-4 mm broad with 3-4 $\mathrm{mm}$ claw, free, elliptic or spathulate with rounded tip. Androgynophore 1-24 mm long. Stamens 6, free; filaments purple, 1-2 cm long; anthers linear, 2-3 mm long. Gynophore 1-2 cm long, extending to $3.5 \mathrm{~cm}$ in fruits. Ovary sessile among stamens or on up to $2 \mathrm{~cm}$ long gynophore, oblong-cylindric, $2.5-4 \times 0.5 \mathrm{~mm}$, elongating after fertilization, green or purplish green, shortly glandular-pubescent; style short, 1-1.2 mm long; stigma capitate, depressed at apex, purple; ovules many, on parietal placentae. Fruits capsule, linear to cylindric, 4-15 cm long, 3-5 mm wide, tapering at both ends; stipes 4-5 cm long. Seeds 1$1.5 \mathrm{~mm}$ in diameter, black-brown, numerous, small, with short irregular cross-ribs and inconspicuous longitudinal ribs. Flowering and fruiting: Throughout the year.

Chromosome number: 2n = 30 (Fedorov 1969).

Ecology: Grows along roadsides, edges of rice fields and sandy river banks.

Specimens examined: Dhaka: Nilkhet-Palashi, 6 x 1978, Mahbuba Halim 290 (DACB). Gaibandha: Palashbari Upazila, Jangalpur, 6 v 1988, Mia et al. M. 1839 (DACB). Kushtia: Chuadanga-Gokulkhali, 2 i 1976, Huq et al. H. 1747 (DACB), Chuadanga-Kalabari, 2 i 1976, Huq et al. H. 1806 (DACB). Rajshahi: Godagari, on the way to Nawabganj, 14 xii 1972, A.M. Huq 744 (DACB).

Geographical distribution: Sri Lanka to southeast and east Asia, Malesia, Africa (type) and America.

Economic importance: Decoction of root is given in fever. Leaves are used in rheumatism by rural people in India (Bakshi 1984).

3. Cleome hassleriana Chodat., Bull. Herb, Boiss. 6, App. 1: 12 (1898). Khan et al., J. Asiatic Soc. (Sc.) 4 (1\&2): 77-79 (1978); Grierson \& Long, Fl. Bhut. 1(2): 416 (1984); Raghavan in Balakrishnan (ed.), Fl. Ind. 2: 320 (1993).

(Plate 3)

English names: Spider flower, Spider plant.

Erect, perennial herb; stem up to $1.5 \mathrm{~m}$ tall. Stem glandular-pubescent, rather hispidulous near the apex, occasionally spiny, longitudinally grooved. Leaves 5-7 foliate, the terminal 3-5 leaflets usually larger up to $7 \times 2 \mathrm{~cm}$, the shorter two lateral ones up to $2.5 \times 1.0 \mathrm{~cm}$, lanceolate to elliptic-lanceolate, acuminate, entire, often ciliolate, glandular-pubescent, often spines on midrib beneath. Petiole $6 \mathrm{~cm}$ long, bearing a pair of short spine-like stipules at base. Racemes 30-40 cm long. Bracts 10-20 × 6-12 mm, simple, ovate, acuminate to acute, pubescent. Pedicel $2-5 \mathrm{~cm}$ long, glandular pubescent. Sepals 4, 8-10 × $2 \mathrm{~mm}$, linear-lanceolate, reflexed, glandular-pubescent, valvate, united at the base, green. Petals 4, free, claw slender, 8-12 mm long, limb ovate, 1.2-1.8 cm, rosy- 
pink, gradually turning into white. Androgynophore $3 \mathrm{~mm}$ long; stamens 6, equal, filaments 4.0-4.5 cm long, anthers oblong, 6-9 mm long. Ovary 5-10 mm long, gynophore at first c. $3 \mathrm{~cm}$, later 5-7.5 cm long; ovules many, on parietal placentae; style none; stigma capitate. Capsule 4.5-9 $\times 0.3 \mathrm{~cm}$, elongated-cylindrical, glandularpubescent, greyish at maturity. Seeds many, c. $2.0 \times 1.2 \mathrm{~mm}$, reniform, yellow. Flowering and fruiting: January-May.

Ecology: Grows in waste places near human habitation and on the roadside, low land.

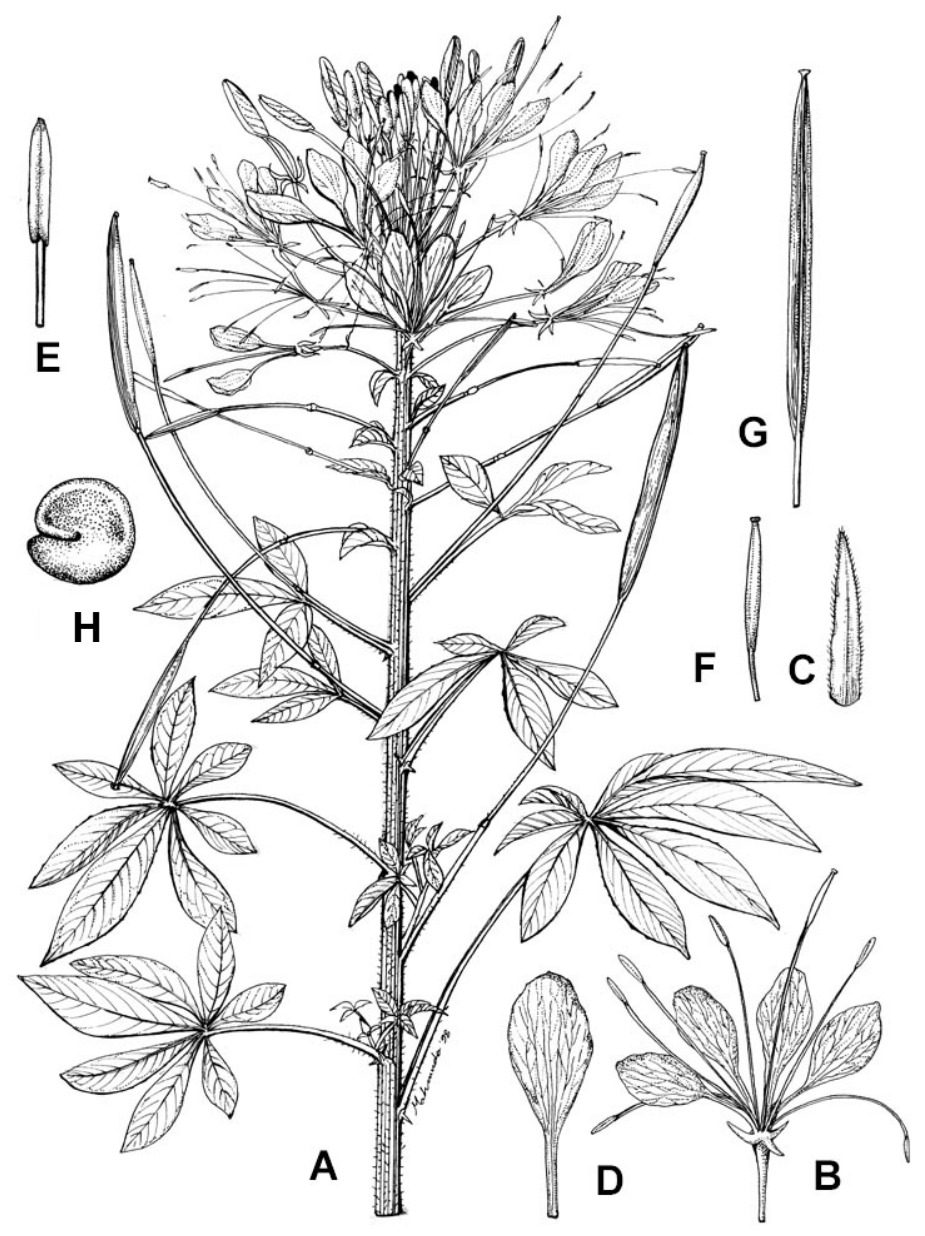

Plate 3. Cleome hassleriana. A. habit $(\times 0.4)$; B. flower $(\times 0.5)$; C. sepal $(\times 2)$; D. petal $(\times 1)$; E. stamen $(\times 0.4)$; F. gynophore $(\times 0.25)$; G. fruit $(\times 0.5)$; H. seed $(\times 6)$.

Specimens examined: Chittagong: Chittagong, 28 v 1978, Yusuf 163 (DACB). Dhaka: Kaoran Bazar, 2 v 1978, A.M. Huq 3816 (DACB); BARC compound, 22 iv 1997, Hosne Ara 23, 24 (DACB). Kishoreganj: Karimganj Jungle Bari village, 13 iii 1988, Mia \& Mahfuz M. 1651 (DACB). Sylhet: Khadimnagar Tea Estate, 13 iv 1978, 
Mia, Huq \& G. Zaman M. 1401 (DACB); Salutikar Airport area, 8 iv 1988, Mahfuz, Huq, Momtaz \& Hosne Ara Mz. 158 (DACB). Sunamganj: Chhatak, 5 i 1978, Huq \& Rahman H. 3642 (DACB); Sunamganj to Dabarghat, 30 i 1979, A.M. Huq 4159 (DACB); Sunamganj, 30 v 1998, Hosne Ara 25, 26 (DACB).

Geographical distribution: Native of tropical South America. Indigenous to Brazil, Argentina and Paraguay.

Economic importance: Cultivated for its showy flowers as an ornamental plant in Bangladesh and other tropical countries.

4. Cleome rutidosperma DC., Prodr, 1: 241 (1824). Jacobs, Fl. Males. 1 (6): 104 (1960); Iltis, Brittonia 12: 290 (1960); Back. \& Bakh. f., Fl. Java 1: 183 (1963); Khan et al., J. Asiatic Soc. (Sc.) 4 (1\&2): 75-77 (1978); Mukherjee in Indian For. 95: 237 (1969); Raghavan in Balakrishnan (ed.), Fl. Ind. 2: 313 (1993); C. ciliata Schum. \& Thonn., Dansk. Vid. Selsk. Afh. 4: 67 (1828).

(Plate 4)

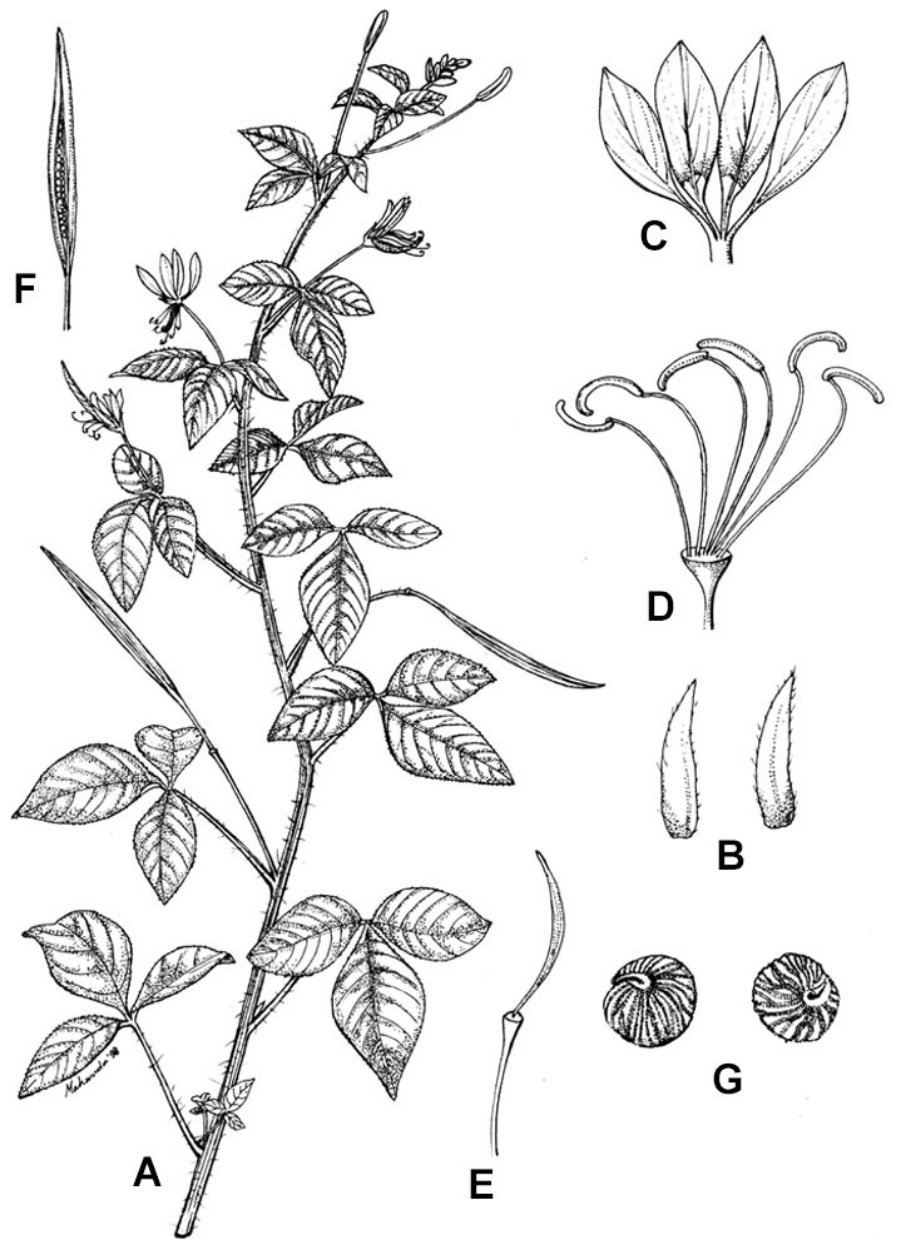

Plate 4. Cleome rutidosperma. A. habit (× 0.4); B. sepals $(\times 4)$; C. petals $(\times 2)$; D. androecium $(\times 2)$; E. gynoecium $(\times 1.5)$; F. fruit $(\times 0.5)$; G. seeds $(\times 6)$. 
English name: Fringed spider flower.

Annual herb, $60 \mathrm{~cm}$ long with a tap root, widely branched, semi-erect to trailing; all parts with scattered, soft, whitish hairs. Stem cylindrical, erect or prostrate, with pricklelike, softish appendages, up to $2 \mathrm{~mm}$ long. Leaflets 3, middle leaflet larger, 3-5 × 1.5-2 $\mathrm{cm}$, the lateral leaflets smaller, up to $2.3 \times 1 \mathrm{~cm}$, alternate, exstipulate, ovate-rhomboid to elliptic, entire, acuminate, base cuneate; nerves 6-8 pairs. Petiole 3-3.5 cm. long, hirsute. Racemes with reduced leaves. Bract leaf like. Flowers violet, bisexual, hypogynous; buds oblong, c. $4 \mathrm{~mm}$ long. Pedicels filiform, 1-3 cm long. Sepals $4,4 \times 1.5 \mathrm{~mm}$, free, linear to linear lanceolate. Petals 4, 8-11 × 2.5-3 mm, free, obovate, clawed, all on the posterior side, purplish, gradually becoming white. Stamens 6, free, the 2 anterior c. $8 \mathrm{~mm}$ long and the 4 posterior ones c. $5 \mathrm{~mm}$ long, all incurved towards the posterior side; filaments 5-10 mm long; anthers 1.5-2 mm long, oblong, greyish, dehiscence longitudinal. Gynophore c. 1.5-2 mm long, elongating to $8 \mathrm{~mm}$ in fruits. Ovary linear, 5-12 mm long, slightly curved, superior, 1-locular with many ovules on two parietal plancentae; style absent; stigma sessile, capitate. Capsule 4.5-6 × 0.3-0.4 cm with parallel veins, glabrous, linear, cylindrical; stipes 2.5-3 cm long. Seeds many, 1.3-1.9 × 1.0-1.5 mm, deep reddish brown, with conspicuous, \pm anastomosing cross-veins, glabrous ribs connected by much weaker longitudinal ribs; a whitish elaiosome present. Flowering and fruiting: Throughout the year.

Chromosome number: 2n= 20 (Kumar and Subramaniam 1986).

Ecology: Grows in waste places, as wayside weed, sometimes on rocks.

Specimens examined: Chittagong: Foy's lake, 9 viii 1989, Huq et al. H. 9327 (DACB); Chittagong, 10 vi 1978, Yusuf 169 (DACB). Cox's Bazar: Moheshkhali, 5 iii 1978, Annanda Kumar Poddar 76 (DACB). Dhaka: Mirpur, Technical, 2 vi 1975, Mokles s.n. (DACB); Curzon Hall campus, 1 vi 1977, M.A. Hassan (DUH). Rangamati: Rangamati, 1 v 1977, Huq \& Rahman H. 3323 (DACB); Near Rangamati College, 25 vi 1987, A.M. Huq \& M.K. Mia H. 8438 (DACB). Rangpur: Saidpur Railway Officer's colony, 15 x 1976, Huq et al. H. 2608 (DACB).

Geographical distribution: Native in south-east Asia, southern, eastern and central Africa, central America, Philippines and Indonesia, or Native to west tropical Africa (type), introduced to Burma and Malesia.

5. Cleome viscosa L., Sp. Pl. 2: 672 (1753). Hook. f. \& Thoms., Fl. Brit. Ind. 1: 170 (1872); Prain, Beng. Pl. 1: 225 (1903), Rep. ed. 1: 149 (1963); Dunn in Gamble, Fl. Pres. Madras: 41: 29 (1915); Kanjilal et al., Fl. Assam 1: 72 (1934); Jacobs, Fl. Males. 1 (6): 103 (1960); Grierson \& Long, Fl. Bhut. 1 (2): 416 (1984); Raghavan in Balakrishnan (ed.), Fl. Ind. 2: 318 (1993); Polanisia viscosa DC., Prod. 1: 242 (1824).

(Plate 5)

Bangla names: Halde hurhure, Hurhuria 
English names: Asian spider flower, Tickweed.

Erect, annual herb, 30-90 cm high, all parts \pm densely brownish glandular pubescent and viscid throughout. Leaves 3 or 5 foliolate, 1.5-4.5 cm long, 1-1.5 cm wide, obovate or ovate, base cuneate, apex acute or obtuse, margin entire, nerves 5-6 pairs, exstipulate. Petiole 1.5-6 cm long. Racemes lax, few-flowered, corymbose up to $30 \mathrm{~cm}$ long. Bracts subsessile, leaf-like, trifoliate. Flowers yellow, actinomorphic. Pedicels 1-2 cm long, elongating up to $4 \mathrm{~cm}$ in fruits. Sepals $4,6-7 \times 1-2 \mathrm{~mm}$, free, lanceolate or oblong, acuminate, glabrous inside, glandular hairy outside. Petals 4 , subequal, free, obovate or

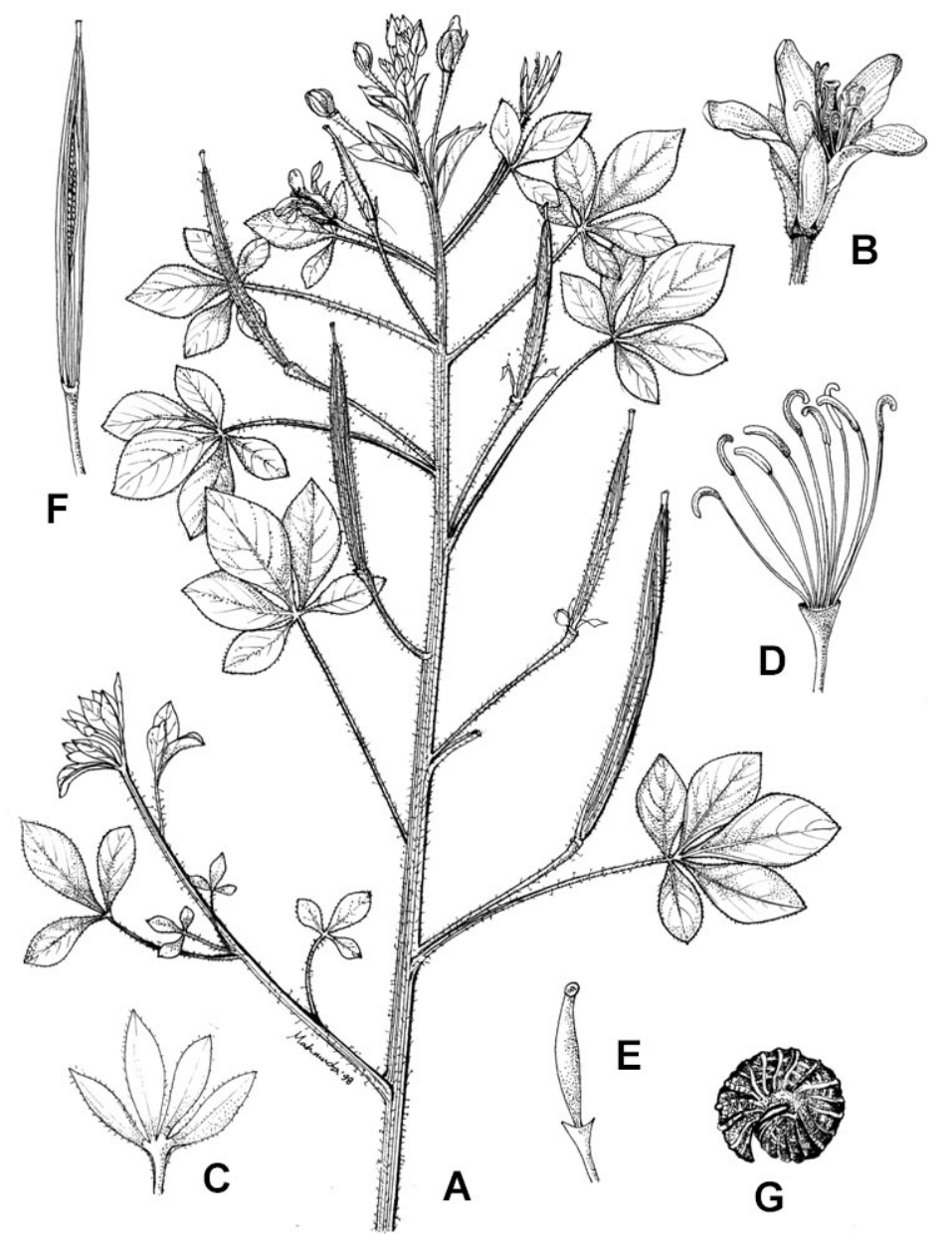

Plate 5. Cleome viscosa. A. habit $(\times 0.4)$; B. flower $(\times 2)$; C. sepals $(\times 2)$; D. androecium $(\times 2)$; E. gynoecium $(\times 1.4)$; F. fruit $(\times 0.5)$; G. seed $(\times 7)$.

oblanceolate to oblong-spathulate, base cuneate with up to $6 \mathrm{~mm}$ long claw at base, rounded at tip, 7-12 × 3-5 mm, yellow, glabrous, distinctly veined. Stamens many, free, glabrous; filaments almost filiform, 3-8 mm long; anthers linear, 1-3 mm long. 
Gynophore absent. Ovary sessile, 3-10 mm long, 2-4 mm wide, oblong-cylindric or linear-oblong, beaked, glandular-pubescent; style 2-6 mm long, slender; stigma usually on a very short style, capitate. Capsule 3-10 cm long, 2-4 mm wide, cylindric, striate, glandular pubescent, narrowed at the tip with distinct veins, hairy; stipes 1-3 mm long. Seeds 1.3-1.8 mm in diameter, red-brown, with narrow cleft, strong cross-ribs and weak concentric ribs. Flowering and fruiting: Throughout the year.

Chromosome number: $2 \mathrm{n}=20$ (Fedorov 1969).

Ecology: Common in waste places, railway tracks, along roadsides and other open and shady places.

Specimens examined: Bagerhat: Mongla (other side of the river), 28 vi 1986, A.M. Huq \& M.K. Mia H. 7808 (DACB). Chittagong: Sitakund, Chandranath Hill, 22 vi 1979, Mia \& Rahman M. 88 (DACB). Cox's Bazar: Teknaf Upazila, Nayapara, 8 vi 1988, Mia et al. M. 1954 (DACB). Comilla: Salban biher, Moinamati, Lalmai, 8 viii 1988, Mahfuz \& A.M. Huq MZ. 225 (DACB). Dhaka: Mohakhali area, 19 vii 1995, Hosne Ara HA. 17, 18, 19 (DACB); Mirpur Botanic Garden, 15 xii 1979, Mia et al. M. 199 (DACB). Dinajpur: Singra, 15 i 1974, M.S. Khan \& A.M. Huq K. 3640 (DACB). Faridpur: Goalando, 16 i 1981, Mia et al. M. 517 (DACB). Jessore: Rupganj, 29 viii 1983, Huq et al. H. 5980 (DACB). Khulna: Near Newsprint Mill area, 29 vi 1973, A. M. Huq 1041 (DUH). Khagrachhari: Matiranga (22 km north), 26 vi 1985, Huq \& Mia H. 7166 (DACB). Kushtia: Chuadanga-Gokerkhali, 2 i 1976, Huq \& Mia H. 1755 (DACB). Kishoreganj: Kishoreganj, 12 iii 1968, Paritosh 26 (DUH); Dhanchira (11/2 miles off), 4 iii 1983, Huq et al. H. 6486 (DACB). Nilphamari: Saidpur near Railway Officer's colony, 15 x 1976, Huq et al. H. 2608 (DACB). Patuakhali: Ferry-ghat, 11 iii 1982, Rahman \& Mia R. 1266 (DACB). Rajshahi: Rajshahi University campus, 19 xi 1988, Huq et al. H. 8786 (DACB). Rangamati: Manikchhari, 24 vi 1987, A.M. Huq \& M.K. Mia H. 8381 (DACB). Satkhira: Sonabaria, 2 v 1984, Khan et al. K. 6625 (DACB). Sunamganj: Chhatak, 5 i 1978, Huq \& Rahman H. 3658 (DACB). Tangail: Mirzapur, 27 vi 1965, K. Begum 39 (DUH).

Geographical distribution: Native in the tropical and warmer parts of India, and the rest of the world.

Economic importance: The seeds are anthelmintic (Bakshi 1984).

\section{Acknowledgements}

The authors would like to thank the authorities and staff members of the following herbaria for allowing to use the herbarium and library facilities: British Natural History Museum (BM); Central National Herbarium (CAL); Bangladesh National Herbarium (DACB) and Dhaka University Herbarium (DUH). Mrs. Mahmuda Akhter, Artist-cumIllustrator, Bangladesh National Herbarium deserves thanks for helping the authors in drawing all the illustrations of the paper. 


\section{References}

Bakshi, D.N.G. 1984. Flora of Murshidabad District, West Bengal, Scientific Publications, Jodhpur, India, pp. 55.

Datta, R.B. and Mitra, J.N. 1953. Common Plants in and around Dacca city. Bull. Bot. Soc. Beng. 7(1\&2): 14.

Fedorov, A.A. 1969. Chromosome numbers of flowering plants. Academy of Sciences of U.S.S.R., Moscow, pp. 926.

Heinig, R.L. 1925. List of Plants of Chittagong Collectorate and Hill Tracts. The Bengal Government Branch press, Darjeeling, India, pp. 84.

Hook. f. and Thomson. 1872. Capparidaceae. In: Flora of British India. Vol. 1: 167-180. (Reprint 1973). Bishen Singh Mahendra Pal Singh, Dehra Dun, India.

Jacobs, M. 1960. Capparidaceae. In: Flora Malesiana series 1, Vol. 6(1): 61-105. Printed in the Netherland.

Kanjilal, U.K., Kanjilal, P.C. and Das, A. 1934. Flora of Assam, Vol. 1 (reprint 1982). A Von Book Company, India, pp. 386.

Khan, M.S. and Banu, F. 1972. A taxonomic report on the angiospermic flora of Chittagong Hill Tracts-2. J. Asiatic Soc. Bangladesh 17 (2): 59-88.

Khan, M.S., Hassan, M.A. and Huq, A.M. 1978. New angiospermic records for Bangladesh-12, Cleome rutidosperma DC. \& Cleome hassleriana Chodat. J. Asiatic Soc. Bangladesh (Sc.) 4(1\&2): 75-79.

Khan, M.S., Rahman, M.M., Huq, A.M., Mia, M.M. K. and Hassan, M. A. 1994. Assessment of biodiversity of Teknaf Game Reserve in Bangladesh focussing on economically and ecologically important plant species. Bangladesh J. Plant Taxon. 1(1): 21-33.

Kumar, V. and Subramaniam, B. 1986. Chromosome atlas of flowering plants of the Indian subcontinent, Vol. 1 (Dicotyledons). Botanical Survey of India, pp. 464.

Mia, M.M.K. and Khan, B. 1995. First list of angiospermic taxa of Bangladesh not included in Hooker’s ‘Flora of British India’ and Prain’s ‘Bengal Plants’. Bangladesh J. Plant. Taxon. 2(1\&2): 25-45.

Prain, D. 1903. Bengal Plants. Vol 1. Indian reprint (1981). Bishen Singh Mahendra Pal Singh, Dehra Dun, India, pp. 663.

Raghavan, R.S. 1993. Capparaceae. In: Sharma, B.D. and Balakrishnan, N.P. (eds.). Flora of India 2. Botanical Survey of India, Calcutta, pp. 248-335.

Rahman, M.A. and Uddin, S.B. 1997. Angiospermic flora of Sitakund in Chittagong, Bangladesh. Bangladesh J. Plant Taxon. 4(1): 17-36.

Rahman, M.O. 2004a. Second list of angiospermic taxa of Bangladesh not included in Hooker's 'Flora of British India’ and Prain’s ‘Bengal Plants’: Series I. Bangladesh J. Plant Taxon. 11(1): 77-82.

Rahman, M.O. 2004b. Second list of angiospermic taxa of Bangladesh not in Hooker's 'Flora of British India’ and Prain’s ‘Bengal Plants’: Series II. Bangladesh J. Plant Taxon. 11(2): 49-56.

Rahman, M.O. and Hassan, M.A. 1995. Angiospermic flora of Bhawal National Park, Gazipur, Bangladesh. Bangladesh J. Plant Taxon. 2(1\&2): 47-79.

Rashid, M.H., Rahman, E. and Rahman, M.A. 2000. Additions to the angiospermic flora of the Moheskhali Island, Cox's Bazar. Bangladesh. Bangladesh J. Plant Taxon. 7(1): 43-63.

Sinclair, J. 1955. Flora of Cox’s Bazar, East Pakistan. Bull. Bot. Soc. Bengal 9(2): 84-116.

Uddin, S.N., Khan, M.S., Hassan, M.A. and Alam, M.K. 1998. An annotated checklist of angiospermic flora of Sita Pahar at Kaptai in Bangladesh. Bangladesh J. Plant Taxon. 5(1): 13-46.

Yusuf, M. 1989. Clemoe diffusa Banks ex DC. A new angiospermic record for Bangladesh. J. Asiatic Soc. Bangladesh (Sc.) 15(2): 149-151. 